\title{
WORKSHOP ON EUROPEAN BEHAVIOURAL INDICATIONS FOR MEN WHO HAVE SEX WITH MEN
}

\author{
Editorial team (eurosurveillance@ecdc.europa.eu) ${ }^{1}$ \\ 1. European Centre for Disease Prevention and Control, Stockholm, Sweden
}

HIV and sexually transmitted infections (STI) reported among men having sex with men (MSM) have risen in western Europe in recent years. This is thought to be a result of unsafe sexual practices, but data in this field are lacking. Many European Union (EU) Member States conduct surveys or hand out questionnaires about sexual behaviour and attitudes, either to patients visiting STI clinics, or in at-risk communities, such as MSM. However, the methods of such behavioural surveillance vary greatly between countries, making it difficult to compare data and obtain an overall view of the current sexual behaviour and attitudes in the EU.

Behavioural surveillance of MSM is usually conducted by posing questions that indicate unsafe sexual practices. Awareness campaigns aimed at the public and health professionals can then target the practices identified. However, there is currently no standard set of indicators for behavioural surveillance of MSM in the EU. The indicators of United Nations General Assembly Special Session (UNGASS) indicators [1], which every country should monitor and report, are not tailored to western European countries and do not specifically address the MSM community. During a workshop held by the Robert Koch Institute in Berlin, Germany, in February 2007 [2], experts from across the EU had stressed the need to harmonise behavioural indicators among countries performing behavioural surveys among MSM, so that national epidemiologists and social scientists can compare results with their European colleagues and target prevention interventions more effectively. On 20 and 21 February 2008, the European Centre for Prevention Disease and Control (ECDC) hosted a technical workshop in Stockholm, Sweden, bringing together epidemiologists and social scientists from across the EU in order to work towards this goal.

The ECDC recently awarded a contract to the University of Lausanne, Switzerland, to analyse behavioural surveillance related to STI and HIV. This study will look at the EU population as a whole as well as specific groups at risk (including MSM), and will result in a proposal for the implementation of harmonised behavioural surveillance in EU Member States. This workshop's outcomes and recommendations will be included in the University of Lausanne's wider project.

The workshop opened with presentations of the results of behavioural surveys among MSM that had been conducted in different countries and regions across western Europe in recent years. Unsurprisingly, the results and methodologies were widely different. Some countries had been collecting data since as far back as 1987, while others were much more recent; some countries are about to start behavioural surveillance in MSM; some countries collected data every year, others sporadically; some countries had wide samples, others very small ones, and so on. Despite the differences, many countries had observed an increase in unsafe sexual practices in recent years.

Two working groups met to discuss a possible set of indicators that could be included in questionnaires across the EU. They were invited to address the following areas: practice of anal intercourse, condom use, history of STI, number of sexual partners, HIV serostatus and HIV testing behaviour. The first group proposed seven key indicators, and the second group proposed eight. There was a great deal of discussion about the nuances and level of detail, but there seemed to be general agreement that surveys should aim to ask respondents the following points:

- The number of sexual partners they had had in the past six months or year;

- Whether they had used a condom the last time they had had anal intercourse;

- Whether they had been diagnosed with an STI in the last year;

- Their HIV sero-status; and

- The venues in which they had usually met partners (saunas, bars, night clubs, online, etc).

Other suggestions included asking about the sero-status of sexual partners, if known; whether or not the respondent is in a relationship, and if so if it is open or closed; the perception of the risk of certain sexual practices; and the ability to express sexual preferences to others. Some delegates felt that there should be a set of core indicators for all countries, and an enhanced set for countries that wish to implement more detailed studies.

Broader issues discussed included how frequently such surveys should be done; how widely; and in what settings, as well as how to recruit respondents. A full report of the meeting is being prepared for publication on the ECDC's website, and the University of Lausanne will consider the recommendations of the workshop in its further activities addressing the groups most at risk, including MSM.

\section{References}

1. United Nations General Assembly Special Session on HIV/AIDS. Monitoring the Declaration of Commitment on HIV/AIDS. Guidelines on Construction of Core Indicators. Available from: http://www.who.int/hiv/strategic/me/en/ isbn9291732389.pdf 
2. Velasco E. Behavioural surveillance and HIV prevention in men who have sex with men: Reports from Australia, Belgium, Canada, France, Germany, Switzerland, the United Kingdom, and the United States. Available from: http://www.wzb.eu/bal/ph/publikationen.en.htm

This article was published on 10 April 2008.

Citation style for this article: Editorial team. Workshop on European behavioural

indications for men who have sex with men. Euro Surveill, 2008:13(15):pii=18853.

Available online: http://www.eurosurveillance.org/ViewArticle.aspx?ArticleId=18853 